\title{
GEOMETRIC PROGRAMMING SOLUTION OF SECOND DEGREE DIFFICULTY FOR CARBON EJECTION CONTROLLED RELIABLE SMART PRODUCTION SYSTEM
}

\author{
Andreas Se Ho Kugele ${ }^{1} \odot$, Waqas Ahmed $^{2} \odot$ and Biswajit Sarkar ${ }^{1,3, *} \odot$
}

\begin{abstract}
Smart manufacturing systems should always aim to be fully sustainable while simultaneously being as reliable as possible which is difficult to reach. Furthermore, climate change especially by carbon emission in the industry is a significant topic and carbon emission should be controlled and reduced to save the environment. Contributing towards a greener environment in a positive manner is done by reducing the number of insufficient items that are produced in a smart production system which also can be reached with higher reliability in the system. Therefore, this study models a smart reliable production system with controlled carbon ejection. To solve the proposed smart production system in this study, a geometric programming approach with a degree of difficulty level two is used which results in optimum results that are quasi-closed. Furthermore, numerical experiments are conducted to validate the proposed model and prove that by using a higher degree geometric programming approach, an optimal solution is found. The numerical results do not only show optimal solutions but also that the smart production system with controlled carbon ejection is reliable.
\end{abstract}

Mathematics Subject Classification. 90B05, 90B06.

Received October 13, 2021. Accepted February 21, 2022.

\section{INTRODUCTION}

Smart production systems should always aim to reduce waste and be as environmentally friendly as possible. A positive contribution towards a greener environment in a smart production system may be inter alia gained by reducing the amount of waste that the manufacturing system is producing as well as controlling the carbon emission ejection. Furthermore, by making the smart production system reliable and sustainable, a great overall positive output can be gained. A smart production system is said to be reliable if there are no defective items. In addition, to call a smart production system (SPS) sustainable, the SPS must fulfill the pillars of sustainability.

Keywords. Smart production system, reliability, geometric programming, setup reduction, controlled carbon ejection.

1 Department of Industrial Engineering, Yonsei University, 50 Yonsei-ro, Sinchon-dong, Seodaemun-gu, Seoul 03722, South Korea.

2 Department of Operations and Supply Chain, NUST Business School, National University of Sciences and Technology, Islamabad 44000, Pakistan.

3 Center of Transdisciplinary Research (CFTR), Saveetha Dental College, Saveetha Institute of Medical and Technical Sciences, Saveetha University, 162, Poonamallee High Road, Velappanchavadi, Chennai 600077, Tamil Nadu, India

*Corresponding author: bsbiswajitsarkar@gmail.com.

(c) The authors. Published by EDP Sciences, ROADEF, SMAI 2022 
Hence, the smart production system must contribute towards a greener environment and bring economic as well as social benefits.

This study considers a smart reliable production system by using a reliability factor and also considering carbon emission ejection. To solve the study, a geometric programming approach is used. In geometric programming (GP), the degree of difficulty has great importance since it displays if there are no solutions, a unique solution, or many solutions. The degree of difficulty in the GP approach in the proposed reliable smart production system of this study equals two which indicates that there are many solutions. By executing numerical examples, it is found that it is possible to find a solution while operating with a higher degree of difficulty than one.

Furthermore, by using geometric programming, the optimum total cost can be found before finding the optimum of the four decision variables that are used which are production rate, reliability, production quantity, and setup cost under managed carbon ejection. In the following, the novelty of the proposed study is displayed:

- The proposed study develops a smart production system under consideration of controlled carbon emission to contribute to the prevention of excessive carbon emission of the manufacturing house which is needed for saving the environment as well as reducing health issues caused by intoxicated air.

- The proposed smart production system is developed in a reliable and sustainable manner. By maintaining a high degree of reliability and sustainability, the production of waste is reduced which contributes to a greener environment.

- Setting up a smart reliable production system is considered to be very expensive. Investments to reduce the expenditure for setup are implemented in the proposed model.

- The study is solved using a posynomial geometric programming method of second degree. Using the second degree geometric programming approach, the optimum cost is found before finding optimum values of decision variables and the study proves that it is possible to find a unique solution utilizing the second degree geometric programming methodology.

This study is structured as follows: Section 2 covers the reviewed literature and Section 3 explains the complete model development of the smart reliable production system with managed carbon ejection. Section 4 deals with the geometric programming solution procedure in which before all else, the global minimum total cost expression is determined. Afterward, the optimum expressions of the four decision variables are determined. Section 5 validates the proposed model of a smart reliable production system with numerical examples and proves that a unique solution can be found while having a degree of difficulty equalling two. Additionally, a sensitivity analysis is conducted in Section 6. Section 7 covers the managerial insights and the last section concludes this study.

\section{Literature REVIEW}

Generally being able to control and reduce carbon emission ejection in smart production systems is inevitable to contribute to a greener environment. Production systems become more and more automated and show development towards smart production systems which is discussed by Kusiak [19]. Also, it is found that for changing a production system into a reliable production system, carbon emission ejection must be considered [29].

\subsection{Smart production system}

A smart production process within a supply chain model with optimal energy consumption was studied by Bhuniya et al. [4]. In the case of a production enterprise, a methodology for developing it into a smart and sustainable production enterprise with sensing is developed by Chavarría-Barrientos et al. [7]. Furthermore, an increasement in energy efficiency in a smart production system by controlling peripheral equipment is done by Bermeo-Ayerbe et al. [3]. Alavian et al. [1] developed a programmable production advisor that is able to find the current status of the system and improvements of a smart production system. 
Also, a smart production system under a SCM is recently developed by Dey et al. [9] in which the lead time is controllable and safety stock, as well as planned backorder, have been considered. Furthermore, a setup cost reduction has been integrated. Relevant factors that decide the implementation of digital technologies as well as information of smart production is studied by Ghobakhloo [13]. The study of Sarkar et al. [27] covers a smart production system for a multi-type biofuel production. Their developed production system is sustainable and includes the implementation of renewable energies.

\subsection{Reliability}

Reliability can inter alia be defined by carbon emission and defectiveness of produced items. A way of reducing carbon emission is to implement smart energy systems. Important technologies and current developments of those smart systems and their impact on carbon emission are shown in a study by Zhu et al. [34]. Habib et al. [14] proved a minimum total expenditure in a supply chain model in which the carbon ejection is also minimized under uncertain conditions. In a previous study of Wang et al. [31], it can be seen that carbon emission ejection influences the judgment of manufacturing and remanufacturing. Sepehri et al. [28] developed an inventory model with joint pricing in which carbon emissions are controlled.

Mahapatra et al. [23] introduced a continuous review production inventory system in which the preparation time for manufacturing is considered to be flexible and the expenditure for setup considered to be dependent on time. Additionally, a fuzzy environment has been assumed for the demand and time of setup. Ullah et al. [30] developed a sustainable supply chain model in which remanufacturing as well as the return of items have been considered. A production system that is not reliable has been discussed in the study of Nahas [25]. Chen et al. [8] proposed an evaluation strategy that evaluates the reliability in a production system with numerous states. Furthermore, Wu and Cui [32] analyzed the reliability of systems that are periodically investigated.

A closed-loop supply chain model that simultaneously considers a conventional and green production under consideration of carbon emission from various sources and considering a stochastic demand is developed by Jauhari et al. [17]. Furthermore, Sarkar et al. [26] discussed inter alia carbon emission ejection and their effects in a SCM that is sustainable and an improvement of production quality in the case of items with defined lifetimes. A vague model with expenditure reliability of an incorporated manufacturing transportation supply chain is developed by Asim et al. [2] which is solved by numerous goal optimizations. Leung [20] found an optimum total cost of an economic production quantity model that considers reliability. In his study, he used a GP approach with a first degree of difficulty to find ideal decision variable values as well as the total expenditure.

\subsection{Geometric programming}

A standard inventory model with various constraints is solved by El-Wakeel and Salman [11] with a geometric programming approach, yet reliability has not been considered. Liu [21] used a geometric programming approach with a degree of difficulty of zero to find the optimum profit as a global result. In a further step, Liu [22] used the concept of a signomial geometric programming approach in which the optimum profit is derived with the usage of interval coefficients and a quantity discount. Furthermore, a robust geometric programming approach is done in a study by Chassein and Goerigk [6].

Geometric programming can also be done in a fuzzy manner. Hence, Cao and Wang [5] discussed the development of uncertain geometric programming. A power optimization of a digital circuit with process variations and time constraints by using fuzzy geometric programming is found by Ghavami et al. [12]. Dressler et al. [10] considered positive certificates build on accumulations of positive circuit polynomials and geometric programming which contains constraints for a polynomial optimization and combines those concepts. Jafarian et al. [16] proposed a programming approach consisting of two steps to solve a nonlinear problem with numerous objectives. The programming approach of the study combines geometric programming and fuzzy optimization techniques. 
TABLE 1. Authors contribution table.

\begin{tabular}{|c|c|c|c|c|c|}
\hline Author(s) & Production system & Reliability & $\begin{array}{l}\text { Carbon } \\
\text { emission }\end{array}$ & $\begin{array}{l}\text { Geometric } \\
\text { programming }\end{array}$ & $\begin{array}{l}\text { Setup } \\
\text { cost }\end{array}$ \\
\hline Leung [20] & $\mathrm{EPQ}$ & $\begin{array}{l}\text { Production } \\
\text { process }\end{array}$ & NA & Degree $=1$ & Variable \\
\hline Liu $[21]$ & NA & NA & NA & Degree $=0$ & NA \\
\hline Liu [22] & NA & NA & NA & Signomial & NA \\
\hline Moon et al. [24] & Constant & $\begin{array}{l}\text { Production } \\
\text { process }\end{array}$ & Considered & Considered & Variable \\
\hline Habib et al. [14] & NA & NA & Considered & NA & NA \\
\hline Chavarría-Barrientos [7] & Smart and sustainable & NA & NA & NA & NA \\
\hline Jafarian et al. $[16]$ & NA & NA & NA & Considered & NA \\
\hline Kusiak [19] & Smart & NA & NA & NA & $\mathrm{NA}$ \\
\hline Tiwari et al. [29] & Sustainable & NA & Considered & NA & NA \\
\hline Wang et al. [31] & Constant & NA & Considered & NA & $\mathrm{NA}$ \\
\hline Asim et al. [2] & Hybrid & Cost & NA & NA & Reduced \\
\hline Cao and Wang [5] & $\mathrm{NA}$ & NA & NA & Fuzzy & NA \\
\hline Chassein and Goerigk [6] & NA & NA & NA & Robust & NA \\
\hline Dressler et al. $[10]$ & NA & NA & NA & Considered & NA \\
\hline El-Wakeel et al. [11] & NA & NA & NA & Considered & NA \\
\hline Ghavami et al. [12] & NA & NA & NA & Fuzzy & NA \\
\hline Yadav et al. [33] & Smart & NA & Considered & NA & Reduced \\
\hline Ullah et al. [30] & Constant & NA & Considered & NA & Considered \\
\hline Alavian et al. $[1]$ & Smart & NA & NA & NA & NA \\
\hline Ghobakhloo [13] & Smart & NA & NA & NA & NA \\
\hline Nahas [25] & Production line & Unreliable & NA & NA & $\mathrm{NA}$ \\
\hline Sepehri et al. [28] & NA & NA & Considered & NA & NA \\
\hline Bhuniya et al. [4] & Smart & NA & NA & NA & Constant \\
\hline Bermeo-Ayerbe et al. [3] & Smart & NA & NA & NA & NA \\
\hline Chen et al. [8] & Multi-state & $\begin{array}{l}\text { Product } \\
\text { quality }\end{array}$ & $\mathrm{NA}$ & NA & $\mathrm{NA}$ \\
\hline Dey et al. [9] & Smart & System & NA & NA & Reduced \\
\hline Mahapatra et al. [14] & Constant & NA & NA & NA & $\begin{array}{l}\text { Time } \\
\text { depen- } \\
\text { dent }\end{array}$ \\
\hline Hayhoe et al. [15] & NA & NA & NA & Posynomial & NA \\
\hline Jauhari et al. [17] & Hybrid & NA & Considered & NA & Constant \\
\hline Kalaiarasi et al. [18] & Multi-item EPQ & NA & Considered & Fuzzy & NA \\
\hline Sarkar et al. $[26]$ & Sustainable & NA & Considered & NA & Reduced \\
\hline Wu and Cui $[32]$ & NA & System & NA & NA & NA \\
\hline Sarkar et al. $[27]$ & Sustainable, smart & NA & Considered & NA & Reduced \\
\hline Zhu et al. [34] & NA & Energy & Considered & NA & NA \\
\hline This study & Smart & $\begin{array}{l}\text { Production } \\
\text { process }\end{array}$ & Considered & Degree $=2$ & $\begin{array}{l}\text { Variable, } \\
\text { Reduced }\end{array}$ \\
\hline
\end{tabular}

Notes. NA is inserted for the case that it is "Not Applicable".

Kalaiarasi et al. [18] developed an economic quantity model with numerous items and solved their model with geometric programming in an uncertain manner using a variety of membership functions for the fuzzification process. Furthermore, Hayhoe et al. [15] used a posynomial geometric programming approach to solve an optimal control problem and find strategies for the best control method. Table 1 displays the authors contribution table. 


\section{MAthematical MODEL}

This section of the study deals with the generation of the model. The mathematical model for this study is divided into a basic problem definition, followed up by notation that are used, assumptions that are made, and the full model development.

\subsection{Problem definition}

Production systems generally tend to produce a significant amount of defective items with an irregular quota of defectiveness and not be fully reliable even if it is a smart production system. Reliability has great importance not only when it comes to the quality of the products and the production system itself but also in terms of waste reduction and having a big positive impact on contributing towards a greener environment.

In a reliable production system, it is possible to reduce waste and have waste control while simultaneously being able to keep the quality of the products that are being produced. A production system that reduces waste instantly contributes to a greener environment. By additionally considering carbon emission ejection, a production system can be further significantly improved.

Therefore, this study deals with a smart reliable production system that considers carbon emission ejection. The goal of this study is to gain a significant improvement of a conventional production system by turning it into a smart production system and using a reliability factor under carbon emission and also obtaining a minimum total cost. To find an optimum production quantity, as well as optimum values for reliability, setup cost, an optimum production rate, and finally an optimum total cost, a geometric programming approach with a positive degree of difficulty is used in this study by which a quasi-closed form of an optimum solution can be found.

\subsection{Notation}

The notation that have been used in this study are the following:

\begin{tabular}{ll}
\hline \multicolumn{2}{l}{ Decision variables } \\
\hline$\kappa$ & Expenditure for setup per setup (\$/setup) \\
$\eta$ & Quota of production (units/unit time) \\
$\delta$ & Manufacturing process reliability \\
$q$ & Production quantity (units/batch) \\
\hline Parameters & \\
\hline$\zeta(\kappa, \delta)$ & Expenditure for depreciation and interest $(\$ /$ year) \\
TC $(\kappa, \eta, \delta, q)$ & Total expenditure of the smart production system $(\$ /$ year) \\
$c_{\mathrm{D}}$ & Development cost function \\
$\alpha$ & Tool cost function \\
$c_{\mathrm{m}}$ & Raw material cost $(\$ /$ unit) \\
$\chi$ & Expenditure for holding $(\$ /$ unit $/$ year) \\
$\tau$ & Demand (units/year) \\
$\xi$ & Scaling factor for investment function \\
$a, b, c$ & Shape parameters for depreciation and interest cost \\
$p$ & Production cost per year $(\$ /$ year) \\
$\omega$ & Setup cost per year $(\$ /$ year) \\
$\Xi$ & Investment cost (\$) \\
$H_{c}$ & Inventory holding cost per year $(\$ /$ year) \\
$\mu_{i}$ & Term expressions for dual function, $i=1, \ldots, 7$ \\
$\varphi_{i}$ & Normalized dual variables, $i=1, \ldots, 7$ \\
$\bar{\varphi}$ & Column vector of normalized dual variables \\
$\phi(\bar{\varphi}, \kappa, q, \delta, \eta)$ & Total expenditure expressed with normalized dual variables \\
$\theta$ & Optimal variables expressed with normalized dual variables \\
$X\left(\varphi_{4}, \varphi_{7}\right)$ & Logarithmic expression of total cost expressed with normalized dual variables \\
\hline
\end{tabular}




\subsection{Assumptions}

The following assumptions are considered for the development of the model in this study.

(1) The considered reliable smart production system only produces one kind of item and each item is inspected. In case a defective item is found at the inspection, the insufficient item is being removed to keep the smart production system reliable.

(2) The reliable smart production system controls carbon emissions, which leads to a reduction of carbon emission ejection and contributes to saving the environment. Each cost that is considered in this study is also considered with managed carbon ejection.

(3) To lower the expenditure for setup, an investment function is considered, which is as follows: $\Xi=\xi \frac{\kappa^{2}}{2}$, where $\xi$ is the scaling parameter. Furthermore, the time boundary is considered to be not existent [33].

(4) The model of this study considers a depreciation and interest cost in which the reliability is considered and is contrarily proportional to the setup cost. The depreciation and interest cost is formulated as follows: $\zeta(\kappa, \delta)=a \kappa^{-b} \delta^{c}$, where the parameters $a, b$ and $c$ are the shape parameters which are constant real numbers that are greater than zero [24].

\subsection{Model development}

\subsubsection{Production cost}

In a reliable smart production system, a production cost should be considered. For the production cost per cycle, the following equation is being used

$$
p=\left(\alpha \eta+\frac{c_{\mathrm{D}}}{\eta}+c_{\mathrm{m}}\right) \frac{\tau}{\delta}
$$

where $\eta$ denotes the variable production quota. The parameter $\alpha$ represents the tool cost function and $c_{\mathrm{D}}$ is the development cost. The parameter $c_{\mathrm{m}}$ represents the expenditure for raw materials.

\subsubsection{Setup cost}

Since this study deals with a production system, considering a setup cost is mandatory. The reliable smart production system is dependant on the reliability of the product since the reliability factor influences the acceptable quality of the product that can satisfy the demand. Therefore, the length of a cycle has to be $\delta q / \tau$. Hence, the expenditure for setup per cycle is as follows

$$
\omega=\frac{\kappa \tau}{q \delta} .
$$

\subsubsection{Investment cost}

Since the setup for a reliable smart production system is very high, a reduction of the setup cost is considered in this study. Therefore, the following investment function is introduced to reduce the setup cost

$$
\Xi=\xi \frac{\kappa^{2}}{2},
$$

where $\xi$ is the scaling parameter.

\subsubsection{Inventory holding cost}

The expenditure for holding inventory is also a mandatory cost that has to be considered in a reliable smart production system. Hence, the expenditure of holding inventory is as follows

$$
H_{c}=\frac{\chi q \delta}{2} .
$$




\subsubsection{Depreciation and interest cost}

Since the decision variable $\delta$ represents the reliability, the unreliability can be written as $1-\delta$ and the depreciation and interest cost can be formulated as the following

$$
\zeta(\kappa, \delta)=a \kappa^{-b}(1-\delta)^{-c_{1}} .
$$

The reliability factor $\delta$ can take values between zero and one. It is impossible for a production process to be perfectly reliable and in case the equation for unreliability is being used and if $\delta$ goes to 1 , it means that equation (3.5) goes to $\infty$. This would mean that the closed-form optimum solution for this model is not possible to be found.

Hence, the following equation for the depreciation and interest cost is used for the model of the reliable smart production system

$$
\zeta(\kappa, \delta)=a \kappa^{-b} \delta^{c},
$$

where $a, b$ and $c$ are the shape parameters and the following holds: $a, b, c>0$.

\subsubsection{Total cost}

The total cost of the reliable smart production system under controlled carbon emission per production cycle can be found by adding all the previously mentioned costs

Total expenditure with controlled carbon ejection $=$ Production cost + Setup cost

$$
\begin{aligned}
& \text { + Investment cost to reduce the setup cost } \\
& + \text { Inventory holding cost } \\
& + \text { Depreciation and interest cost. }
\end{aligned}
$$

The mathematical formulation of the accumulated expenditure is the following

$$
\begin{aligned}
\mathrm{TC}(\kappa, q, \delta, \eta)= & \tau \kappa q^{-1} \delta^{-1}+\alpha \eta \tau \delta^{-1}+\frac{c_{\mathrm{D}} \tau}{\eta \delta}+c_{\mathrm{m}} \tau \delta^{-1}+\frac{\chi q \delta}{2} \\
& +\tau a \kappa^{-b} q^{-1} \delta^{c-1}+\xi \frac{\kappa^{2}}{2}
\end{aligned}
$$

where $\eta, \kappa, q, \delta>0$.

\section{Geometric programming solution to the mathematical model}

To solve the previously proposed model, a geometric programming approach is used. In geometric programming, an important factor is the degree of difficulty through which one can obtain information if the model has a unique solution, many solutions, or no solutions. The degree of difficulty in this study equals two, which implies that there are many solutions. The aim of this study is to find an optimum minimum cost under carbon emission of the proposed smart production system at a degree of difficulty of two. When it comes to geometric programming, two different categories are existent in which one is called posynomial, and the other is called signomial. Both categories contain power functions but in a signomial problem, one or more coefficients are negative while in a posynomial problem all coefficients have to be positive. By using a geometric programming approach, the optimum minimum cost can be found before calculating the ideal values of the decision variables by finding the optimum values of the dual variables. The following equations are considered to find an optimum solution of this model through geometric programming

$$
\begin{aligned}
& \mu_{1}=\tau \kappa q^{-1} \delta^{-1} \\
& \mu_{2}=\alpha \eta \tau \delta^{-1}
\end{aligned}
$$




$$
\begin{aligned}
\mu_{3} & =\frac{c_{\mathrm{D}} \tau}{\eta \delta} \\
\mu_{4} & =c_{\mathrm{m}} \tau \delta^{-1} \\
\mu_{5} & =\frac{\chi q \delta}{2} \\
\mu_{6} & =\tau a \kappa^{-b} q^{-1} \delta^{c-1} \\
\mu_{7} & =\xi \frac{\kappa^{2}}{2} .
\end{aligned}
$$

The previously mentioned total cost equation can be written in the following form

$$
\begin{aligned}
\operatorname{TC}(\kappa, q, \delta, \eta) & =\mu_{1}+\mu_{2}+\mu_{3}+\mu_{4}+\mu_{5}+\mu_{6}+\mu_{7} \\
& \geq\left(\frac{\mu_{1}}{\varphi_{1}}\right)^{\varphi_{1}}\left(\frac{\mu_{2}}{\varphi_{2}}\right)^{\varphi_{2}}\left(\frac{\mu_{3}}{\varphi_{3}}\right)^{\varphi_{3}}\left(\frac{\mu_{4}}{\varphi_{4}}\right)^{\varphi_{4}}\left(\frac{\mu_{5}}{\varphi_{5}}\right)^{\varphi_{5}}\left(\frac{\mu_{6}}{\varphi_{6}}\right)^{\varphi_{6}}\left(\frac{\mu_{7}}{\varphi_{7}}\right)^{\varphi_{7}} \\
& \equiv \phi(\bar{\varphi}, \kappa, q, \delta, \eta) .
\end{aligned}
$$

Furthermore, the sum of $\varphi_{1}$ to $\varphi_{7}$ must equal to 1 and $\varphi_{1}$ to $\varphi_{7}$ must be greater than 0 .

Equation (4.8) consists of a primal function that is shown on the left side of the equation and a dual function that is displayed on the right-hand side of the function. The column vector $\bar{\varphi}$ consists of the normalized dual variables $\varphi_{1}$ to $\varphi_{7}$. By using the values of $\mu_{1}$ to $\mu_{7}$, the following equation is found

$$
\begin{aligned}
\phi(\bar{\varphi}, \kappa, q, \delta, \eta)= & \left(\frac{\tau \kappa q^{-1} \delta^{-1}}{\varphi_{1}}\right)^{\varphi_{1}}\left(\frac{\alpha \eta \tau \delta^{-1}}{\varphi_{2}}\right)^{\varphi_{2}}\left(\frac{c_{\mathrm{D}} \tau \eta^{-1} \delta^{-1}}{\varphi_{3}}\right)^{\varphi_{3}}\left(\frac{c_{\mathrm{m}} \tau \delta^{-1}}{\varphi_{4}}\right)^{\varphi_{4}} \\
& \times\left(\frac{\chi q \delta}{2 \varphi_{5}}\right)^{\varphi_{5}}\left(\frac{\tau a \kappa^{-b} q^{-1} \delta^{c-1}}{\varphi_{6}}\right)^{\varphi_{6}}\left(\frac{\xi \kappa^{2}}{2 \varphi_{7}}\right)^{\varphi_{7}} \\
= & \left(\frac{\tau}{\varphi_{1}}\right)^{\varphi_{1}}\left(\frac{\alpha \tau}{\varphi_{2}}\right)^{\varphi_{2}}\left(\frac{c_{\mathrm{D}} \tau}{\varphi_{3}}\right)^{\varphi_{3}}\left(\frac{c_{\mathrm{m}} \tau}{\varphi_{4}}\right)^{\varphi_{4}}\left(\frac{\chi}{2 \varphi_{5}}\right)^{\varphi_{5}}\left(\frac{\tau a}{\varphi_{6}}\right)^{\varphi_{6}}\left(\frac{\xi}{2 \varphi_{7}}\right)^{\varphi_{7}} \\
& \times \eta^{\varphi_{2}-\varphi_{3}} \kappa^{\varphi_{1}-b \varphi_{6}+2 \varphi_{7}} q^{-\varphi_{1}+\varphi_{5}-\varphi_{6}} \delta^{-\varphi_{1}-\varphi_{2}-\varphi_{3}-\varphi_{4}+\varphi_{5}+\varphi_{6}(c-1)},
\end{aligned}
$$

in which $\bar{\varphi}$ is arbitrary, which means that the exponents of each decision variable equals to zero.

Hence, equation (4.9) can be written as follows

$$
\phi\left(\varphi_{1}, \varphi_{2}, \varphi_{3}, \varphi_{4}, \varphi_{5}, \varphi_{6}, \varphi_{7}\right)=\left(\frac{\tau}{\varphi_{1}}\right)^{\varphi_{1}}\left(\frac{\alpha \tau}{\varphi_{2}}\right)^{\varphi_{2}}\left(\frac{c_{\mathrm{D}} \tau}{\varphi_{3}}\right)^{\varphi_{3}}\left(\frac{c_{\mathrm{m}} \tau}{\varphi_{4}}\right)^{\varphi_{4}}\left(\frac{\chi}{2 \varphi_{5}}\right)^{\varphi_{5}}\left(\frac{\tau a}{\varphi_{6}}\right)^{\varphi_{6}}\left(\frac{\xi}{2 \varphi_{7}}\right)^{\varphi_{7}} .
$$

The model is accepted when the orthogonality and normality conditions hold. The orthogonality and normality conditions are as follows

$$
\begin{aligned}
\varphi_{2}-\varphi_{3} & =0 \\
\varphi_{1}-b \varphi_{6}+2 \varphi_{7} & =0 \\
-\varphi_{1}+\varphi_{5}-\varphi_{6} & =0 \\
-\varphi_{1}-\varphi_{2}-\varphi_{3}-\varphi_{4}+\varphi_{5}+\varphi_{6}(c-1) & =0 \\
\varphi_{1}+\varphi_{2}+\varphi_{3}+\varphi_{4}+\varphi_{5}+\varphi_{6}+\varphi_{7} & =1 .
\end{aligned}
$$

Furthermore, the degree of difficulty has to be specified to know if geometric programming can be used. The degree of difficulty (DoD) can be found as follows

$$
\text { DoD }=\text { Number of terms }- \text { Number of decision variables }-1
$$




$$
= \begin{cases}\text { unique solution, } & \text { if degree of difficulty }=0 \\ \text { many solutions, } & \text { if degree of difficulty }>0 \\ \text { no solution, } & \text { if degree of difficulty }<0 .\end{cases}
$$

Hence, the degree of difficulty in this model is the following

$$
\text { The degree of difficulty }=7-4-1=2 \text {. }
$$

The solutions of $\varphi_{1}, \varphi_{2}, \varphi_{3}, \varphi_{5}$ and $\varphi_{6}$ in terms of $\varphi_{4}$ and $\varphi_{7}$ are the following

$$
\begin{aligned}
& \varphi_{1}=b\left(\frac{\left(-1-3 \varphi_{7}\right)}{(-2-2 b-c)}\right)-2 \varphi_{7} \\
& \varphi_{2}=\left(1-\varphi_{4}-\left(1-c\left(\frac{\left(-1-3 \varphi_{7}\right)}{(-2-2 b-c)}\right)-\varphi_{7}\right)-(1+b)\left(\frac{\left(-1-3 \varphi_{7}\right)}{(-2-2 b-c)}\right)+\varphi_{7}\right) \frac{1}{2} \\
& \varphi_{3}=\left(1-\varphi_{4}-\left(1-c\left(\frac{\left(-1-3 \varphi_{7}\right)}{(-2-2 b-c)}\right)-\varphi_{7}\right)-(1+b)\left(\frac{\left(-1-3 \varphi_{7}\right)}{(-2-2 b-c)}\right)+\varphi_{7}\right) \frac{1}{2} \\
& \varphi_{5}=1-c\left(\frac{\left(-1-3 \varphi_{7}\right)}{(-2-2 b-c)}\right)-\varphi_{7} \\
& \varphi_{6}=\frac{\left(-1-3 \varphi_{7}\right)}{(-2-2 b-c)} .
\end{aligned}
$$

To maximize the dual function with optimal $\varphi_{4}^{*}$ and $\varphi_{7}^{*}$, a logarithm of the function (4.10) can be used. Hence, the following equation can be obtained

$$
\begin{aligned}
X\left(\varphi_{4}, \varphi_{7}\right)= & \ln \phi\left(\varphi_{4}, \varphi_{7}\right) \\
= & \varphi_{1} \ln \left(\frac{\tau}{\varphi_{1}}\right)+\varphi_{2} \ln \left(\frac{\alpha \tau}{\varphi_{2}}\right)+\varphi_{3} \ln \left(\frac{c_{\mathrm{D}} \tau}{\varphi_{3}}\right)+\varphi_{4} \ln \left(\frac{c_{\mathrm{m}} \tau}{\varphi_{4}}\right)+\varphi_{5} \ln \left(\frac{\frac{\chi}{2}}{\varphi_{5}}\right) \\
& +\varphi_{6} \ln \left(\frac{\tau a}{\varphi_{6}}\right)+\varphi_{7} \ln \left(\frac{\xi \frac{1}{2}}{\varphi_{7}}\right) .
\end{aligned}
$$

From equation (4.10), one can get the following

$$
\begin{aligned}
\mathrm{TC}(\kappa, q, \delta, \eta) & \geq \phi^{*}\left(\varphi_{1}^{*}, \varphi_{2}^{*}, \varphi_{3}^{*}, \varphi_{4}^{*}, \varphi_{5}^{*}, \varphi_{6}^{*}, \varphi_{7}^{*}\right) \\
& =\phi\left(\varphi_{1}^{*}, \varphi_{2}^{*}, \varphi_{3}^{*}, \varphi_{4}^{*}, \varphi_{5}^{*}, \varphi_{6}^{*}, \varphi_{7}^{*}\right) \\
& =\left(\frac{\tau}{\varphi_{1}^{*}}\right)^{\varphi_{1}^{*}}\left(\frac{\alpha \tau}{\varphi_{2}^{*}}\right)^{\varphi_{2}^{*}}\left(\frac{c_{\mathrm{D}} \tau}{\varphi_{3}^{*}}\right)^{\varphi_{3}^{*}}\left(\frac{c_{\mathrm{m}} \tau}{\varphi_{4}^{*}}\right)^{\varphi_{4}^{*}}\left(\frac{\frac{\chi}{2}}{\varphi_{5}^{*}}\right)^{\varphi_{5}^{*}}\left(\frac{\tau a}{\varphi_{6}^{*}}\right)^{\varphi_{6}^{*}}\left(\frac{\xi \frac{1}{2}}{\varphi_{7}^{*}}\right)^{\varphi_{7}^{*}} .
\end{aligned}
$$

Taking the equation (4.24) into consideration, the following equation can be displayed

$$
\begin{aligned}
\operatorname{Min} \operatorname{TC}(\kappa, q, \delta, \eta) & =\operatorname{Max} \phi^{*}\left(\varphi_{1}^{*}, \varphi_{2}^{*}, \varphi_{3}^{*}, \varphi_{4}^{*}, \varphi_{5}^{*}, \varphi_{6}^{*}, \varphi_{7}^{*}\right) \\
& =\phi^{*}\left(\varphi_{1}^{*}, \varphi_{2}^{*}, \varphi_{3}^{*}, \varphi_{4}^{*}, \varphi_{5}^{*}, \varphi_{6}^{*}, \varphi_{7}^{*}\right) .
\end{aligned}
$$

In the following, the global optimum values of the decision variables are found.

$$
\frac{\mu_{1}^{*}}{\varphi_{1}^{*}}=\frac{\mu_{2}^{*}}{\varphi_{2}^{*}}=\frac{\mu_{3}^{*}}{\varphi_{3}^{*}}=\frac{\mu_{4}^{*}}{\varphi_{4}^{*}}=\frac{\mu_{5}^{*}}{\varphi_{5}^{*}}=\frac{\mu_{6}^{*}}{\varphi_{6}^{*}}=\frac{\mu_{7}^{*}}{\varphi_{7}^{*}}=\theta
$$

Using equation (4.8), the following can be obtained

$$
\theta^{\varphi_{1}^{*}+\varphi_{2}^{*}+\varphi_{3}^{*}+\varphi_{4}^{*}+\varphi_{5}^{*}+\varphi_{6}^{*}+\varphi_{7}^{*}}=\theta=\phi^{*} .
$$




$$
\text { Hence, } \mu_{i}^{*}=\varphi_{i}^{*} \phi^{*}, \quad i=1,2,3,4,5,6,7 \text {. }
$$

From equation (4.27), the following applies

$$
\begin{aligned}
\mu_{7}^{*} & =\varphi_{7}^{*} \phi^{*} \\
\text { which implies } \kappa^{*} & =\sqrt{\frac{2 \phi^{*} \varphi_{7}^{*}}{\xi}} .
\end{aligned}
$$

The optimum expression for $\delta$ is as follows

$$
\begin{aligned}
\mu_{4}^{*} & =\varphi_{4}^{*} \phi^{*} \\
\text { and } \quad \delta^{*} & =\frac{c_{\mathrm{m}} \tau}{\phi^{*} \varphi_{4}^{*}} .
\end{aligned}
$$

Furthermore, the optimum production rate is expressed as follows

$$
\begin{gathered}
\mu_{2}^{*}=\varphi_{2}^{*} \phi^{*} \\
\text { which implies } \eta^{*}=\frac{\phi^{*} \varphi_{2}^{*} \delta^{*}}{\alpha \tau}=\frac{\phi^{*} \varphi_{2}^{*}\left(\frac{c_{\mathrm{m}} \tau}{\phi^{*} \varphi_{4}^{*}}\right)}{\alpha \tau},
\end{gathered}
$$

and optimum $q$ is the following

$$
\begin{gathered}
\mu_{1}^{*}=\varphi_{1}^{*} \phi^{*} \\
\text { which leads to } q^{*}=\frac{\tau \kappa^{*}}{\delta^{*} \phi^{*} \varphi_{1}^{*}}=\frac{\tau\left(\sqrt{\frac{2 \phi^{*} \varphi_{7}^{*}}{\xi}}\right)}{\left(\frac{c_{\mathrm{m}} \tau}{\phi^{*} \varphi_{4}^{*}}\right) \phi^{*} \varphi_{1}^{*}} .
\end{gathered}
$$

\section{NumericAl EXAMPLE}

To approve the proposed model of this study, numerical examples have been conducted in this section. Geometric programming is the only methodology in which the optimum total cost can be obtained without obtaining optimum values for decision variables. According to geometric programming theory, each value of optimum $\varphi_{1}^{*}$ to $\varphi_{7}^{*}$ must be greater than zero and the sum of $\varphi_{1}^{*}$ to $\varphi_{7}^{*}$ equals to one. This study conducts numerical examples for two different scenarios to validate the model. The Appendix A contains the Hessian matrices and determinant values for both scenarios of the dual function. $H_{11}$ is in both cases negative and $H_{22}$ is in both cases positive which indicates that the global maximum is found. Referring to equation (4.25), the global maximum of the dual function is the global minimum of the total cost function.

\section{Scenario 1}

Table 2 displays the input data that is used to conduct the numerical experiment of Scenario 1 . The optimum values for $\varphi_{1}, \varphi_{2}, \varphi_{3}, \varphi_{4}, \varphi_{5}, \varphi_{6}, \varphi_{7}$ of Scenario 1 are displayed in Table 3 .

Furthermore, the optimum values of the four decision variables and the optimum cumulated expenditure of Scenario 1 are displayed in Table 4.

Scenario 1 considers that the manufacturer has a demand of 600 units and an inventory holding cost of $5 \$$ per unit. Furthermore, the scaling factor for the investment to reduce the expenditure of setup is taken as 2200 and the material cost is considered to be $0.005 \$$ per unit. Using the input data from Table 2, the optimum values of $\varphi_{1}^{*}$ to $\varphi_{7}^{*}$ can be obtained from which the optimum total expenditure is found. Afterwards, the optimum values of the decision variables are identified. The proposed reliable smart production system in Scenario 1 has a $97 \%$ reliability which is displayed by the parameter $\delta^{*}$. Figure 1 displays the graphical representation of optimization for Scenario 1 using the dual variables $\varphi_{4}$ and $\varphi_{7}$. The maximum of $\phi^{*}\left(\varphi_{1}^{*}, \varphi_{2}^{*}, \varphi_{3}^{*}, \varphi_{4}^{*}, \varphi_{5}^{*}, \varphi_{6}^{*}, \varphi_{7}^{*}\right)$ is the minimum of $\mathrm{TC}(\kappa, q, \delta, \eta)$ (refer to Eq. (4.25)). 
TABLE 2. Input data for numerical example of Scenario 1.

\begin{tabular}{lllllllll}
\hline \hline$a$ & $b$ & $c$ & $\chi(\$ /$ unit $)$ & $\alpha$ & $\tau$ (units $)$ & $\xi$ & $c_{\mathrm{D}}$ & $c_{\mathrm{m}}$ \\
\hline 0.25 & 1.25 & 18.75 & 5 & 0.001 & 600 & 2200 & 40 & 0.005 \\
\hline
\end{tabular}

TABLE 3. Optimum values of $\varphi_{1}$ to $\varphi_{7}$ of Scenario 1 .

\begin{tabular}{lllllll}
\hline \hline$\varphi_{1}^{*}$ & $\varphi_{2}^{*}$ & $\varphi_{3}^{*}$ & $\varphi_{4}^{*}$ & $\varphi_{5}^{*}$ & $\varphi_{6}^{*}$ & $\varphi_{7}^{*}$ \\
\hline 0.00134237 & 0.40859 & 0.40859 & 0.01021475 & 0.0960636 & 0.0466894 & 0.0285097 \\
\hline
\end{tabular}

TABLE 4. Optimum results of Scenario 1.

\begin{tabular}{lllll}
\hline \hline $\mathrm{TC}^{*}$ & $\kappa^{*}$ & $\delta^{*}$ & $\eta^{*}$ & $q^{*}$ \\
\hline 301.971 & 0.08846 & 0.972588 & 200 & 134.638 \\
\hline
\end{tabular}

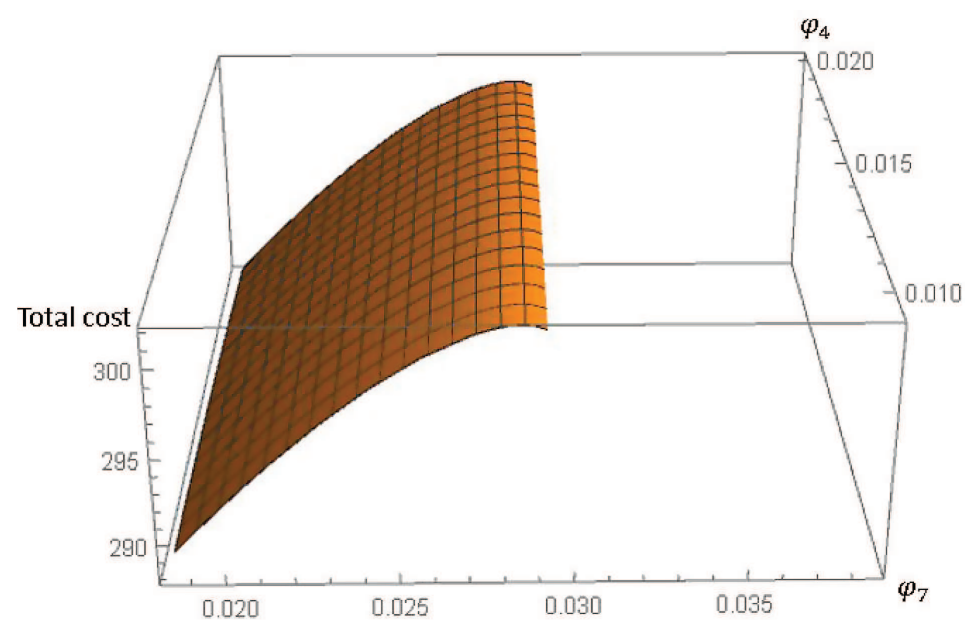

Figure 1. Optimization of Scenario 1.

\section{Scenario 2}

A second scenario of the proposed model is considered to further validate and enhance the effectiveness of the study. Scenario 2 considers a reduced holding cost per unit which is changed to $3 \$$ per unit while the considered demand is increased by 20 units. Furthermore, the development and material cost have been reduced. Additionally, the scaling parameter for the investment function is reduced on a large scale from 2200 to 800 . Table 5 displays the considered input data and Table 6 shows the optimum values of $\varphi_{1}^{*}$ to $\varphi_{7}^{*}$ for Scenario 2 of the proposed model.

Table 7 displays the values for the optimum total expenditure as well as the decision variables. Scenario 2 shows a production quantity of almost 350 units per batch with a production rate of 173 units. Furthermore, the reliability of the considered reliable smart production system in Scenario 2 is $99.8 \%$. Figure 2 graphically shows the optimization for Scenario 2. 
TABLE 5. Input data for numerical example of Scenario 2.

\begin{tabular}{lllllllll}
\hline \hline$a$ & $b$ & $c$ & $\chi(\$ /$ unit $)$ & $\alpha$ & $\tau$ (units) & $\xi$ & $c_{\mathrm{D}}$ & $c_{\mathrm{m}}$ \\
\hline 0.5 & 1.5 & 16.75 & 3 & 0.001 & 620 & 800 & 30 & 0.001 \\
\hline
\end{tabular}

TABLE 6. Optimum values of $\varphi_{1}$ to $\varphi_{7}$ of Scenario 2 .

\begin{tabular}{lllllll}
\hline \hline$\varphi_{1}^{*}$ & $\varphi_{2}^{*}$ & $\varphi_{3}^{*}$ & $\varphi_{4}^{*}$ & $\varphi_{5}^{*}$ & $\varphi_{6}^{*}$ & $\varphi_{7}^{*}$ \\
\hline 0.00105993 & 0.401515 & 0.401515 & 0.0023181 & 0.104521 & 0.0512005 & 0.0378704 \\
\hline
\end{tabular}

TABLE 7. Optimum results of Scenario 2.

\begin{tabular}{lllll}
\hline \hline $\mathrm{TC}^{*}$ & $\kappa^{*}$ & $\delta^{*}$ & $\eta^{*}$ & $q^{*}$ \\
\hline 267.818 & 0.159236 & 0.998644 & 173.205 & 348.26 \\
\hline
\end{tabular}

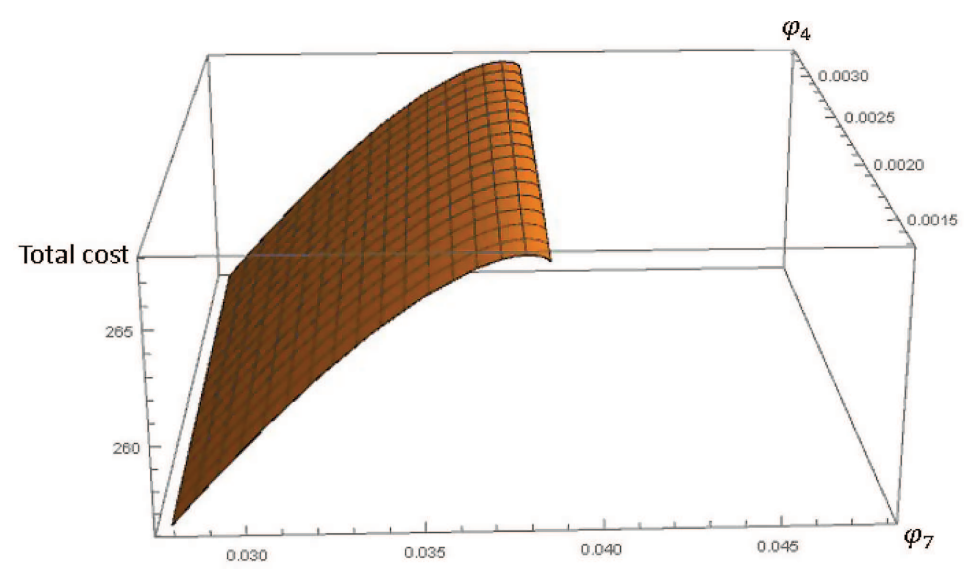

FIGURE 2. Optimization of Scenario 2.

\section{Sensitivity ANALYSis}

The following section discusses the sensitivity of the model towards the input data values. Hence, the sensitivity analysis of Scenario 1 is displayed in Table 8 and the sensitivity analysis of Scenario 2 is shown in Table 9.

For the investigation of sensibility of the first scenario of the proposed model in this study, the influence of changes of the considered cost parameters are inspected. The notation "NA" implicates that changing the cost parameter in the associated manner, it is mathematically not possible in this particular model to find the total cost. In case of highering the tool cost or development cost above $25 \%$, results cannot be given with the used input data and by using geometric programming since the variables $\varphi_{4}$ and $\varphi_{7}$ both come in terms of imaginary numbers. The same result occurs for lowering the unit holding cost to $25 \%$ or lower.

The sensitivity analysis shows that the material $\operatorname{cost} c_{\mathrm{m}}$ does almost not influence the optimum total cost at all by changing its values. The most influential and significantly high changes of the total cost occur by changing the tool cost and development cost. Both costs influence the total cost in the same manner. In case of decreasing 
TABLE 8. Sensitivity analysis of the Scenario 1.

\begin{tabular}{llllll}
\hline \hline Parameters & Changes $(\%)$ & TCD $(\%)$ & Parameters & Changes $(\%)$ & TCD (\%) \\
\hline \multirow{4}{*}{$\chi$} & -50 & NA & & -50 & -32.587 \\
& -25 & NA & $\alpha$ & -25 & -12.453 \\
& +25 & +2.179 & & +25 & NA \\
& +50 & +4.018 & & +50 & NA \\
\hline \multirow{2}{*}{$c_{\mathrm{m}}$} & -50 & -0.514 & & -50 & -32.587 \\
& -25 & -0.256 & $c_{\mathrm{D}}$ & -25 & -12.453 \\
& +25 & +0.255 & & +25 & $\mathrm{NA}$ \\
& +50 & +0.51 & & +50 & $\mathrm{NA}$ \\
\hline
\end{tabular}

Notes. NA is inserted for the case that it is "Not Applicable".

TABLE 9. Sensitivity analysis of Scenario 2.

\begin{tabular}{llllll}
\hline \hline Parameters & Changes $(\%)$ & TCD $(\%)$ & Parameters & Changes $(\%)$ & TCD $(\%)$ \\
\hline \multirow{4}{*}{$\chi$} & -50 & -6.989 & & -50 & NA \\
& -25 & -2.95 & $\alpha$ & -25 & NA \\
& +25 & $\mathrm{NA}$ & & +25 & +9.378 \\
& +50 & $\mathrm{NA}$ & & +50 & +17.696 \\
\hline \multirow{4}{*}{$c_{\mathrm{m}}$} & -50 & -0.112 & & -50 & $\mathrm{NA}$ \\
& -25 & -0.058 & $c_{\mathrm{D}}$ & -25 & $\mathrm{NA}$ \\
& +25 & +0.058 & & +25 & +9.378 \\
& +50 & +0.116 & & +50 & +17.696 \\
\hline
\end{tabular}

Notes. NA is inserted for the case that it is "Not Applicable".

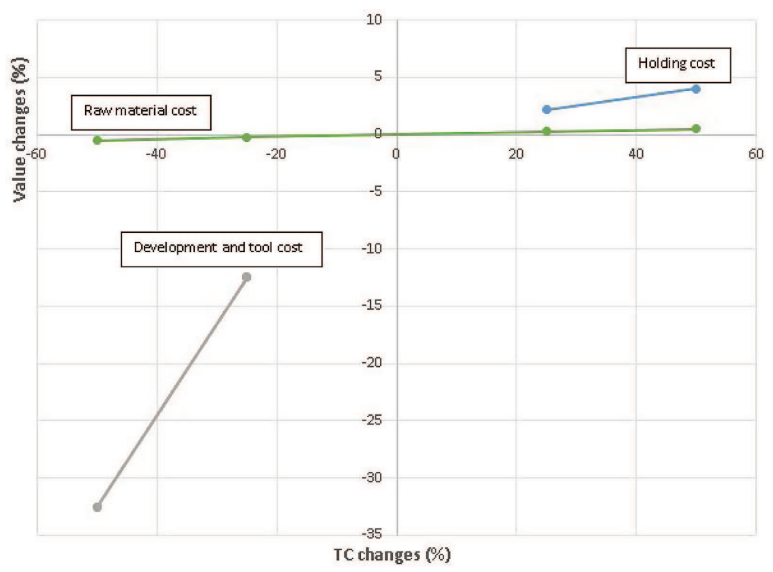

Figure 3. Sensitivity analysis illustration of Scenario 1.

the tool cost or development cost by $25 \%$, the total cost reduces by around $12.5 \%$ and reducing those costs by $50 \%$ results in a total cost reduction of around $32.5 \%$. If the holding cost per unit is increased by $25 \%$ and $50 \%$, the total expenditure increases by approximately $2 \%$ and $4 \%$, respectively. The sensitivity analysis shows that adjusting the tool cost and development cost are the best choices for lowering the total cost due to their high sensitivity impact on the total cost. Figure 3 displays a graphical representation of the sensitivity analysis of Scenario 1. Table 9 displays the sensitivity analysis of Scenario 2 of the proposed model. 


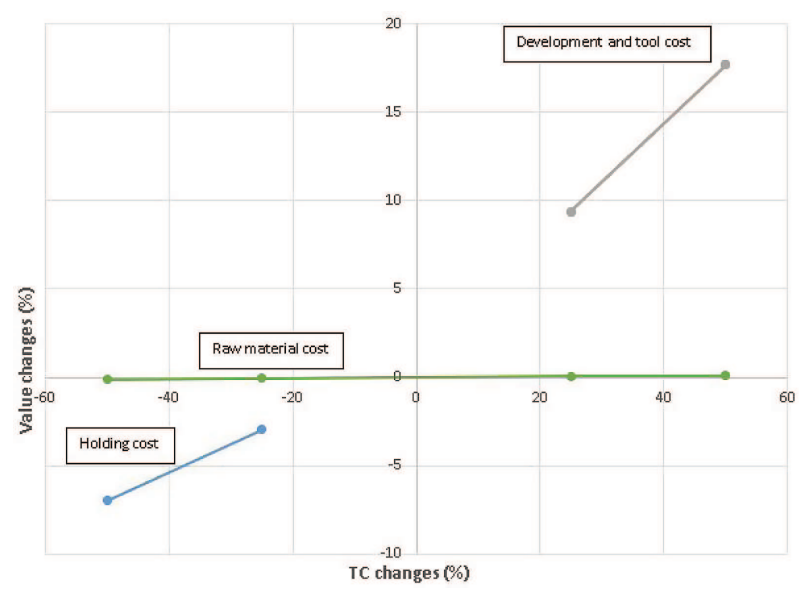

Figure 4. Sensitivity analysis illustration of Scenario 2.

The sensitivity analysis for Scenario 2 shows that similar to Scenario 1, the development cost and tool cost behave in the same manner and have the biggest impact on the total expenditure. Increasing those costs by $25 \%$ and $50 \%$ leads to an increasement of the total expenditure by around $9.4 \%$ and $17.7 \%$, respectively. Decreasing the unit holding cost in Scenario 2 of the model by $25 \%$ reduces the total cost by almost $3 \%$ and decreasing it by $50 \%$ reduces the total cost by around $7 \%$. Changing the material cost almost has no impact on the total expenditure. Figure 4 shows a graphical display of the sensitivity analysis for Scenario 2.

\section{MAnAgerial Insights}

This section discusses the industrial benefits for industry managers and are as follows:

- Implementing a reliable smart production system is necessary to protect the environment and reduce carbon emissions in the industrial sector as well as making the production system more sustainable. Additionally to the positive effects of a greener environment, a smart reliable production system allows the industrial manager to be more responsive to the market environment and enhances the manager's ability to compete in it.

- Considering Figures 3 and 4 of this study, the industrial manager must concentrate on the tool cost as well as development expenditure. The figures show that the two expenditures are highly sensible for reducing as well as increasing the total cost. On the contrary, the industrial manager does not need to focus much on the raw material cost due to its low sensibility and should keep an eye on the inventory holding cost.

- Since the expenditure for setup may be very high, the industrial manager should consider using the discrete investment used in this study to reduce the setup cost.

- The industrial manager should also consider using a geometric programming approach for evaluating changes. Using the geometric programming approach, which this study proves can be done with a second degree of difficulty, the industrial manager can find the total expenditure before finding the optimum values of decision variables. Hence, the industrial manager can find out in a faster manner if and how much beneficial it is to have changes in input parameters without knowing the optimum values of the decision variables yet.

\section{Conclusions}

The model of this study considers a smart production system with controlled carbon ejection that is sustainable. In the development of this study, an investment cost has been included to lower the setup cost of the reliable 
smart manufacturing system. Furthermore, a reliability factor $\delta$ has been used and is seen as a decision variable. The optimum production quantity, as well as setup cost, are found. To solve the proposed model of this study, a geometric programming approach is used. The advantage of using geometric programming as a methodology is that the global optimum total cost can be found before finding the ideal values of the decision variables. When it comes to geometric programming, the degree of difficulty displays if there are no solutions, a unique solution, or many solutions. Thus far in the literature, any model that has been solved with geometric programming either had a degree of difficulty of 0 (unique solution) or a degree of difficulty of 1 (many solutions). Not only is this proposed study developing a smart production system with controlled carbon ejection, but also solves the model via geometric programming that has a degree of difficulty of 2. By conducting numerical examples, the proposed model is validated and a minimum total cost is found. Furthermore, the numerical examples prove that an optimum solution can be found by using a geometric programming approach with a degree of difficulty of 2 . Hence, this study proves that with a degree of difficulty of 2 , geometric programming can be used to solve the model.

The proposed model may be extended in various ways. One way to extend the study may be to further develop the proposed manufacturing system that consists of a singular stage to a numerous-stage manufacturing system or even numerous-stage with numerous cycles. Also, an extension may be done by slightly changing the model and solving it with a higher degree of difficulty than 2 by inter alia using constraints such as a space constraint for storage. To make the proposed smart and reliable production system even more sustainable and have a greater impact on a greener environment, the study can be extended by considering renewable energies or other energy optimization strategies [27]. Furthermore, to make the study closer to reality, fuzziness could be introduced [12]. The proposed model considers a smart production system with controlled carbon emission and uses a posynomial geometric programming approach to solve it. Advancing the smart and reliable production system by inter alia further introducing a holding cost for not only perfect but also insufficient items and a reworking expenditure as well as considering random defective rates during the manufacturing process [27], the proposed study is not able to be solved by geometric programming in a posynomial manner and a signomial geometric programming approach can be used [22].

\section{Appendix A.}

The following displays the Hessian matrices for $\phi^{*}$

$$
\begin{aligned}
& H_{11}=\left(\begin{array}{l}
\left.\partial_{\varphi_{7}, \varphi_{7}} \phi^{*}\right) \\
H_{22}=\left(\begin{array}{l}
\partial_{\varphi_{7}, \varphi_{7}} \phi^{*} \partial_{\varphi_{7}, \varphi_{4}} \phi^{*} \\
\partial_{\varphi_{4}, \varphi_{7}} \phi^{*}
\end{array} \partial_{\varphi_{4}, \varphi_{4}} \phi^{*}\right.
\end{array}\right) .
\end{aligned}
$$

Hessian determinant values

\begin{tabular}{lll}
\hline & $H_{11}$ & $H_{22}$ \\
\hline Scenario 1 & -814285 & $2.437 \times 10^{10}$ \\
Scenario 2 & -852901 & $9.882 \times 10^{10}$ \\
\hline
\end{tabular}

Acknowledgements. This study has been supported by the International Joint Research Grant by Yonsei Graduate School (Support year: 2020 Spring Semester).

\section{REFERENCES}

[1] P. Alavian, Y. Eun, S.M. Meerkov and L. Zhang, Smart production systems: automating decision-making in manufacturing environment. Int. J. Prod. Res. 58 (2020) 828-845.

[2] Z. Asim, S.A. Jalil and S. Javaid, An uncertain model for integrated production-transportation closed-loop supply chain network with cost reliability. Sust. Prod. Consump. 17 (2019) 298-310. 
[3] M.A. Bermeo-Ayerbe, C. Ocampo-Martínez and J. Diaz-Rozo, Adaptive predictive control for peripheral equipment management to enhance energy efficiency in smart manufacturing systems. J. Clean. Prod. 291 (2021) 125556.

[4] S. Bhuniya, S. Pareek, B. Sarkar and B.K. Sett, A Smart Production Process for the Optimum Energy Consumption with Maintenance Policy under a Supply Chain Management. Processes 9 (2021) 19.

[5] B.-Y. Cao and P.-H. Wang, The Origin and Development of Fuzzy Geometric Programming. Fuzzy Inf. Eng. 11 (2019) 203-211.

[6] A. Chassein and M. Goerigk, On the complexity of robust geometric programming with polyhedral uncertainty. Oper. Res. Let. 47 (2019) 21-24.

[7] D. Chavarría-Barrientos, R. Batres, P.K. Wright and A. Molina, A methodology to create a sensing, smart and sustainable manufacturing enterprise. Int. J. Prod. Res. 56 (2018) 584-603.

[8] Z. Chen, Z. Chen, D. Zhou, T. Xia and E. Pan, Reliability evaluation for multi-state manufacturing systems with qualityreliability dependency. Comp. Ind. Eng. 154 (2021) 107166.

[9] B.K. Dey, S. Bhuniya and B. Sarkar, Involvement of controllable lead time and variable demand for a smart manufacturing system under a supply chain management. Exp. Sys. App. 184 (2021) 115464.

[10] M. Dressler, S. Iliman and T.D. Wolff, An approach to constrained polynomial optimization via nonnegative circuit polynomials and geometric programming. J. Symb. Comp. 91 (2019) 149-172.

[11] M.F. El-Wakeel and R.S.A. Salman, Multi-product, multi-venders inventory models with different cases of the rational function under linear and non-linear constraints via geometric programming approach. J. King Saud Univ. Sci. 31 (2019) $902-912$.

[12] B. Ghavami, M. Raji, R. Rasaizadi and M. Mashinchi, Process variation-aware gate sizing with fuzzy geometric programming. Comp. Elec. Eng. $\mathbf{7 8}$ (2019) 259-270.

[13] M. Ghobakhloo, Determinants of information and digital technology implementation for smart manufacturing. Int. J. Prod. Res. 58 (2020) 2384-2405.

[14] M.S. Habib, O. Asghar, A. Hussain, M. Imran, M.P. Mughal and B. Sarkar, A robust possibilistic programming approach toward animal fat-based biodiesel supply chain network design under uncertain environment. J. Clean. Prod. 278 (2021) 122403.

[15] M. Hayhoe, F. Barreras and V.M. Preciado, Multitask learning and nonlinear optimal control of the COVID-19 outbreak: a geometric programming approach. Ann. Rev. Cont. 52 (2021) 495-507.

[16] E. Jafarian, J. Razmi and M.F. Baki, A flexible programming approach based on intuitionistic fuzzy optimization and geometric programming for solving multi-objective nonlinear programming problems. Exp. Sys. with App. 93 (2018) $245-256$.

[17] W.A. Jauhari, I.N. Pujawan and M. Suef, A closed-loop supply chain inventory model with stochastic demand, hybrid production, carbon emissions, and take-back incentives. J. Clean. Prod. 320 (2021) 128835.

[18] K. Kalaiarasi, M.S. Begum and M. Sumathi, Optimization of unconstrained multi-item (EPQ) model using fuzzy geometric programming with varying fuzzification and defuzzification methods by applying python. To appear in: Mat. Tod.: Proc. (2021) DOI: $10.1016 / j$.matpr.2020.10.588.

[19] A. Kusiak, Smart manufacturing. Int. J. Prod. Res. 56 (2018) 508-517.

[20] K.-N.F. Leung, A generalized geometric-programming solution to "An economic production quantity model with flexibility and reliability consideration". Europ. J. Oper. Res. 176 (2007) 240-251.

[21] S.-T. Liu, Profit maximization with quantity discount: an application of geometric programming. Appl. Math. Comp. 190 (2007) 1723-1729.

[22] S.-T. Liu, Using geometric programming to profit maximization with interval coefficients and quantity discount. Appl. Math. Comp. 209 (2009) 259-265.

[23] A.S. Mahapatra, H.N Soni, M.S. Mahapatra, B. Sarkar and S. Majumder, A Continuous Review Production-Inventory System with a Variable Preparation Time in a Fuzzy Random Environment. Mathematics 9 (2021) 747.

[24] I. Moon, W.Y. Yun and B. Sarkar, Effects of variable setup cost, reliability, and production costs under controlled carbon emissions in a reliable production system. Europ. J. Ind. Eng. (2022).

[25] N. Nahas, Buffer allocation, equipment selection and line balancing optimisation in unreliable production lines. Eur. J. Ind. Eng. 14 (2020) 217-246.

[26] B. Sarkar, M. Sarkar, B. Ganguly and L.E. Cárdenas-Barrón, Combined effects of carbon emission and production quality improvement for fixed lifetime products in a sustainable supply chain management. Int. J. Prod. Econ. 231 (2021) 107867.

[27] B. Sarkar, B. Mridha and S. Pareek, A sustainable smart multi-type biofuel manufacturing with the optimum energy utilization under flexible production. J. Clean. Prod. 332 (2022) 129869.

[28] A. Sepehri, U. Mishra, M.-L. Tseng and B. Sarkar, Joint Pricing and Inventory Model for Deteriorating Items with Maximum Lifetime and Controllable Carbon Emissions under Permissible Delay in Payments. Mathematics 9 (2021) 470.

[29] S. Tiwari, Y. Daryanto and H.M. Wee, Sustainable inventory management with deteriorating and imperfect quality items considering carbon emission. J. Clean. Prod. 192 (2018) 281-292.

[30] M. Ullah, I. Asghar, M. Zahid, M. Omair, A. AlArjani and B. Sarkar, Ramification of remanufacturing in a sustainable three-echelon closed-loop supply chain management for returnable products. J. Clean. Prod. 290 (2021) 125609.

[31] X. Wang, Y. Zhu, H. Sun and F. Jia, Production decisions of new and remanufactured products: implications for low carbon emission economy. J. Clean. Prod. 171 (2018) 1225-1243.

[32] B. Wu and L. Cui, Reliability analysis of periodically inspected systems with competing risks under Markovian environments. Comp. Ind. Eng. 158 (2021) 107415. 
[33] D. Yadav, R. Kumari, N. Kumar and B. Sarkar, Reduction of waste and carbon emission through the selection of items with cross-price elasticity of demand to form a sustainable supply chain with preservation technology. J. Clean. Prod. 297 (2021) 126298.

[34] H. Zhu, H.H. Goh, D. Zhang, T. Ahmad, H. Liu, S. Wang, S. Li, T. Liu, H. Dai and T. Wu, Key technologies for smart energy systems: Recent developments, challenges, and research opportunities in the context of carbon neutrality. J. Clean. Prod. 331 (2022) 129809 .

\section{Subscribe to Open (S20) A fair and sustainable open access model}

This journal is currently published in open access under a Subscribe-to-Open model (S2O). S2O is a transformative model that aims to move subscription journals to open access. Open access is the free, immediate, online availability of research articles combined with the rights to use these articles fully in the digital environment. We are thankful to our subscribers and sponsors for making it possible to publish this journal in open access, free of charge for authors.

\section{Please help to maintain this journal in open access!}

Check that your library subscribes to the journal, or make a personal donation to the $\mathrm{S} 2 \mathrm{O}$ programme, by contacting subscribers@edpsciences.org

More information, including a list of sponsors and a financial transparency report, available at: https://www. edpsciences.org/en/maths-s2o-programme 